\title{
Bayesian Integrated Data Analysis of Fast-Ion Measurements by Velocity-Space Tomography
}

Salewski, M.; Nocente, M.; Jacobsen, A.S.; Binda, F.; Cazzaniga, C.; Eriksson, J.; Geiger, B.; Gorini, G.; Hellesen, C.; Kiptily, V. G.

Total number of authors:

21

Published in:

Fusion Science and Technology

Link to article, DOI:

$10.1080 / 15361055.2017 .1380482$

Publication date:

2018

Document Version

Peer reviewed version

Link back to DTU Orbit

Citation $(A P A)$ :

Salewski, M., Nocente, M., Jacobsen, A. S., Binda, F., Cazzaniga, C., Eriksson, J., Geiger, B., Gorini, G., Hellesen, C., Kiptily, V. G., Koskela, T., Korsholm, S. B., Kurki-Suonio, T., Leipold, F., Moseev, D., Nielsen, S. K., Rasmussen, J., Schneider, P. A., Sharapov, S. E., ... Tardocchi, M. (2018). Bayesian Integrated Data Analysis of Fast-lon Measurements by Velocity-Space Tomography. Fusion Science and Technology, 74(1-2), 23-36. https://doi.org/10.1080/15361055.2017.1380482

\section{General rights}

Copyright and moral rights for the publications made accessible in the public portal are retained by the authors and/or other copyright owners and it is a condition of accessing publications that users recognise and abide by the legal requirements associated with these rights.

- Users may download and print one copy of any publication from the public portal for the purpose of private study or research.

- You may not further distribute the material or use it for any profit-making activity or commercial gain

- You may freely distribute the URL identifying the publication in the public portal 


\title{
Bayesian integrated data analysis of fast-ion measurements
}

\author{
by velocity-space tomography
}

M. Salewski, ${ }^{\mathrm{a}, *}$ M. Nocente, ${ }^{\mathrm{b}, \mathrm{c}}$ A.S. Jacobsen, ${ }^{\mathrm{d}}$ F. Binda, ${ }^{\mathrm{e}}$ C. Cazzaniga, ${ }^{\mathrm{f}}$ J. Eriksson, ${ }^{\mathrm{e}}$

B. Geiger, ${ }^{\mathrm{d}}$ G. Gorini, ${ }^{\text {b,c }}$ C. Hellesen, ${ }^{\mathrm{e}}$ V.G. Kiptily, ${ }^{\mathrm{g}}$ T. Koskela, ${ }^{\text {,i }}$ S.B. Korsholm, ${ }^{\mathrm{a}}$

T. Kurki-Suonio, ${ }^{\text {h }}$ F. Leipold, ${ }^{a}$ D. Moseev, ${ }^{\mathrm{j}}$ S.K. Nielsen, ${ }^{\mathrm{a}}$ J. Rasmussen, ${ }^{\mathrm{a}}$ P.A. Schneider ${ }^{\mathrm{d}}$

S.E. Sharapov, ${ }^{g}$ M. Stejner, ${ }^{a}$ M Tardocchi, ${ }^{c}$ JET Contributors*

the ASDEX Upgrade team ${ }^{\dagger}$ and the EUROfusion MST1 team ${ }^{\ddagger}$

${ }^{a}$ Technical University of Denmark, Department of Physics, Kgs. Lyngby, Denmark

${ }^{b}$ University of Milano Bicocca, Department of Physics, Milano, Italy

${ }^{c}$ Istituto di Fisica del Plasma, Consiglio Nazionale delle Ricerche, Milano, Italy

${ }^{d}$ Max-Planck-Institut für Plasmaphysik, Garching, Germany

${ }^{e}$ Department of Physics and Astronomy, Uppsala University, Sweden

${ }^{f}$ ISIS Facility, Science and Technology Facilities Council, Rutherford Appleton Laboratory, Didcot, UK

${ }^{g}$ CCFE, Culham Science Centre, Abingdon, Oxon, UK

${ }^{h}$ Aalto University, Department of Applied Physics, Aalto, Finland

${ }^{i}$ NERSC, Lawrence Berkeley National Laboratory, Berkeley, CA, USA

${ }^{j}$ Max-Planck-Institut für Plasmaphysik, Greifswald, Germany

*Technical University of Denmark, Dep. of Physics, Fysikvej, Building 309,

DK-2800 Kgs. Lyngby, Denmark, tel. +45 236684 44, msal@fysik.dtu.dk

Number of pages: 32

Number of tables: 0

Number of figures: 8 


\title{
Bayesian integrated data analysis of fast-ion measurements by velocity-space tomography
}

\author{
M. Salewski, M. Nocente, A.S. Jacobsen, F. Binda, C. Cazzaniga, J. Eriksson, \\ B. Geiger, G. Gorini, C. Hellesen, V.G. Kiptily, T. Koskela, S.B. Korsholm, T. Kurki- \\ Suonio, F. Leipold, D. Moseev, S.K. Nielsen, J. Rasmussen, P.A. Schneider, S.E. Shara- \\ pov, M. Stejner, M Tardocchi, JET Contributors ${ }^{\mathrm{a}}$, the ASDEX Upgrade team ${ }^{\mathrm{b}}$ and \\ the EUROfusion MST1 team ${ }^{\mathrm{c}}$
}

\begin{abstract}
Bayesian integrated data analysis combines measurements from different diagnostics to jointly measure plasma parameters of interest such as temperatures, densities, and drift velocities. Integrated data analysis of fast-ion measurements has long been hampered by the complexity of the strongly non-Maxwellian fast-ion distribution functions. This has recently been overcome by velocity-space tomography. In this method $2 \mathrm{D}$ images of the velocity distribution functions consisting of a few hundreds or thousands of pixels are reconstructed using the available fast-ion measurements. Here we present an overview and current status of this emerging technique at ASDEX Upgrade and JET based on fast-ion D-alpha spectroscopy, collective Thomson scattering, gamma-ray and neutron emission spectrometry and neutral particle analyzers. We discuss Tikhonov regularization within the Bayesian framework. The implementation for different types of diagnostics as well as the uncertainties are discussed, and we highlight the importance of integrated data analysis of all available detectors.
\end{abstract}

Keywords: tokamaks, fast ions, tomography, velocity-space tomography

\footnotetext{
${ }^{\text {a }} \mathrm{See}^{1}$

${ }^{\mathrm{b}} \mathrm{See}^{2}$

${ }^{\mathrm{c}} \mathrm{See}^{3}$
} 


\section{INTRODUCTION}

Integrated data analysis refers to the combined analysis of measurements from several different detectors or diagnostics to jointly infer parameters of interest..,5 The inference of the plasma temperature, densities or drift velocities by integrated data analysis relies on the assumption of Maxwellian distributions of the particles. However, the ion distributions in fusion plasmas are usually not in thermal equilibrium, but are non-Maxwellian due to the intense plasma heating. For example, velocity distribution functions in plasmas heated by neutral beam injection (NBI) can have peaks due to energetic ion sources at full, half and one-third NBI energy at a pitch determined by the geometry. The pitch is defined as $p=v_{\|} / v$ where $v_{\|}$is velocity component parallel to the magnetic field and $v$ the velocity magnitude. Electromagnetic wave heating in the ion cyclotron range of frequencies (ICRF) causes anisotropy because ICRF heating increases the velocity components perpendicular to the magnetic field, often into the MeV-range. Early modelling assumed bi-Maxwellian distribution functions with different temperatures parallel and perpendicular to the magnetic field, but modern ICRF codes predict features that are not well described by bi-Maxwellians in many heating scenarios. For example, a cut-off energy of the velocity distribution function is predicted in deuterium ICRF heating at the third harmonic. ${ }^{6}$ It would often be an oversimplification to model such velocity distribution functions by analytic formulas. The lack of simple models has hampered integrated data analysis of fast-ion measurements in the past.

Integrated data analysis of fast-ion measurements from at least two detectors has recently become possible by velocity-space tomography. ${ }^{7-20}$ The fast-ion velocity distribution function is described by a $2 \mathrm{D}$ image consisting of a few hundreds or thousands of pixels. The amplitudes in these pixels are then determined by the avail- 
able measurement data and any prior information one might have by a regularized least-squares fit.

Experimentally, the method was introduced based on ASDEX Upgrade $^{21}$ data first using three simultaneously measured spectra from fast-ion $\mathrm{D}_{\alpha}$ (FIDA) spectroscopy in three different views observing the plasma center. ${ }^{10,11}$ Now four to five

simultaneously measured FIDA spectra are routinely used. ${ }^{12-19} \mathrm{NBI}^{10-18}$ as well as ICRF heated plasmas ${ }^{19}$ have been studied, including sawtoothing plasmas ${ }^{12-18}$ and NBI startup scenarios. ${ }^{18}$ First movies of fast-ion velocity distribution functions at ASDEX Upgrade have been presented. ${ }^{18}$ Integrated data analysis by combining different fast-ion diagnostics ${ }^{9}$ has been experimentally demonstrated for FIDA and collective Thomson scattering (CTS) measurements at ASDEX Upgrade ${ }^{15,17}$ and very recently for $\gamma$-ray spectrometry (GRS) and neutron emission spectrometry (NES) at JET. ${ }^{20}$

This paper is organized as follows. In section II. we describe the velocity-space tomography method using Tikhonov regularization with prior information, and in section III. this approach is related to the Bayesian framework. In section IV. we discuss so-called weight functions, which are analogous to lines-of-sight in positionspace tomography, of the most common fast-ion diagnostics. Section V. deals with uncertainties. Section VI. highlights the importance of integrated data analysis of the available measurements. An outlook is presented in section VII., and section VIII. concludes the paper.

\section{HIGH DEFINITION VELOCITY-SPACE TOMOGRAPHY}

In analogy to position-space tomography, the forward model to compute synthetic fast-ion measurements from a fast-ion velocity distribution function is cast as 
the matrix equation ${ }^{8}$

$$
\widetilde{S}=\widetilde{W} F
$$

where $F$ is the fast-ion velocity distribution function written as a vector, $\widetilde{S}$ contains the measurements written as another vector, and $\widetilde{W}$ is a matrix holding the forward model. The calculation of $\widetilde{W}$ is discussed in section IV.. To take measurement uncertainty $\widetilde{\sigma}_{S}$ into account, each entry in $\widetilde{S}$ is normalized with its associated uncertainty, and so is the corresponding line in $\widetilde{W}{ }^{9}$ Hence we obtain the normalized matrix equation

$$
S=W F
$$

in the normalized quantities $S$ and $W$. The tomography problem now is to find a stable and useful solution for $F$, given $S$ and $W$. As the signal-to-noise ratio $S$ is on the same order of magnitude for most diagnostics, whereas the measured signal $\widetilde{S}$ can differ by orders of magnitudes depending on the units, the normalization by $\widetilde{\sigma}_{s}$ substantially improves the conditioning of the tomography problem and allows integrated data analysis of measurements from different diagnostics.

Equation 2 has no exact solution due to noise in the measurements, and hence we instead seek to find a best estimate. However, as in most tomography problems, it turns out that $W$ is ill-conditioned, so that small changes in $S$ result in large changes in $F$ in a standard least-squares fit. The measurement noise therefore generates random jitter in the inversion, and no stable inversion can be found. To date velocity-space tomography has relied on standard inversion methods: truncated singular value decomposition, the maximum entropy method, and a few variants of the Tikhonov regularization. ${ }^{10-12,14,18}$ We here discuss Tikhonov's regularization 
method and solve the minimization problem

$$
F^{*}=\arg \min _{F}\left\|\left(\begin{array}{c}
W \\
\lambda L
\end{array}\right) F-\left(\begin{array}{c}
S \\
0
\end{array}\right)\right\|_{2} \text { subject to } F^{*} \geq 0
$$

where we also demand the solution to be non-negative. ${ }^{18}$ The upper row minimizes the two-norm of the residual of equation 2. Tikhonov's formulation adds the lower row to the least-squares fit which penalizes large values of the two-norm of $\lambda L F$. $L$ is a matrix representing an operator on $F$ such that $L F$ quantifies an undesired property of the solution. If $L$ is the identity matrix, $\lambda L F$ penalizes large amplitudes of $F$. If $L$ is a numerical gradient operator, $\lambda L F$ penalizes large gradients and hence seeks out smooth solutions. The regularization parameter $\lambda$ balances the goodness-of-fit and the regularization requirements and must be found as part of the solution.

Compared with many other tomography applications, the amount of measured fast-in diagnostic data is small due to the limited optical access to the plasma and the often comparatively low signal-to-noise ratio. Therefore inversions often have socalled artifacts which are spurious features of the inversion. Artifacts can be reduced by installing additional fast-ion diagnostics ${ }^{8,14}$ and by optimizing the discharges for signal-to-noise ratio. For example, FIDA works very well in L-mode plasmas with low density and few impurities. ${ }^{10,12-14}$

High-definition tomography techniques additionally make use of prior information to remedy artifacts and to improve the inversions. ${ }^{18}$ Besides the usual demand on smoothness, cogent prior information is the non-negativity of the velocity distribution function, the measured absence of evidence for fast ions within the detection limit, and the position of the injection sources in velocity space for NBI scenarios. Further, a numerical simulation can be used as prior knowledge of the velocity dis- 
tribution function. With these types of prior information, equation 3 is written in extended form as ${ }^{18}$

$F^{*}=\arg \min _{F}\left\|\left(\begin{array}{c}W \\ \lambda \kappa L\end{array}\right) F-\left(\begin{array}{c}S \\ \lambda \kappa L F_{\text {sim }}\end{array}\right)\right\|_{2}$ subject to $\left\{\begin{array}{l}F^{*} \geq 0 \\ F^{*}\left(v_{\| 0}, v_{\perp 0}\right)=0\end{array}\right.$

Here $\kappa=\kappa\left(v_{\|}, v_{\perp}\right)$ encodes the velocity-space positions of the particle sources of the NBI at the full, half and one-third NBI injection energies, $F^{*}\left(v_{\| 0}, v_{\perp 0}\right)=$ 0 is the velocity-space region with negligible fast-ion densities according to nullmeasurements, and $F_{\text {sim }}$ is a numerical simulation used as prior information. These high-definition tomography techniques improved results for the five-view FIDA diagnostic at the tokamak ASDEX Upgrade and further allow the use of inversion techniques for more common FIDA systems with two or three views. The nonnegativity constraint is also essential for tomographic inversion at JET. ${ }^{20}$

\section{BAYESIAN FRAMEWORK FOR VELOCITY-SPACE TOMOG- RAPHY WITH TIKHONOV REGULARIZATION}

This section justifies the velocity-space tomography formalism using Tikhonov regularization in the Bayesian picture and clarifies the connection between the regularization and the prior information. Bayes' theorem links the posterior distribution function with the likelihood function and the prior distribution function:

$$
\operatorname{prob}(F \mid S)=\frac{\operatorname{prob}(S \mid F) \times \operatorname{prob}(F)}{\operatorname{prob}(S)}
$$

Here the posterior $\operatorname{prob}(F \mid S)$ is the probability of the amplitudes of the pixel values describing the distribution function, given the normalized data. The prior $\operatorname{prob}(F)$ is the probability of the distribution function before considering the normalized data. The likelihood function $\operatorname{prob}(S \mid F)$ is the probability of the normalized data, given 
the distribution function. Lastly, $\operatorname{prob}(S)$ is the probability of the normalized data, often called the evidence. Using the forward model, we can assign probabilities to the likelihood function:

$$
\operatorname{prob}(S \mid F) \propto \exp \left(-\frac{1}{2}(\widetilde{W} F-\widetilde{S})^{T} \widetilde{\Sigma}_{S}(\widetilde{W} F-\widetilde{S})\right)
$$

where $\widetilde{\Sigma}_{S}$ is the covariance matrix for the data $\widetilde{S}$. Assuming the noise to be independent and normally distributed, the likelihood function reduces to

$$
\operatorname{prob}(S \mid F) \propto \exp \left(-\frac{1}{2}\|W F-S\|_{2}^{2}\right)
$$

Recall that $W$ and $S$ are normalized by the uncertainties. We further assume the prior distribution to be a multivariate Gaussian:

$$
\operatorname{prob}(F) \propto \exp \left(-\frac{1}{2}\left(F-F_{0}\right)^{T} C^{-1}\left(F-F_{0}\right)\right)
$$

where $C$ is the covariance matrix for $F_{0} . F_{0}$ is the estimate of the velocity distribution function before considering the data. If a numerical simulation $F_{\text {sim }}$ is available, we may set $F_{0}=F_{\text {sim }}$. Since the covariance matrix is positive semidefinite, so is its inverse $C^{-1}$. Hence we can introduce the symmetric decomposition

$$
C^{-1}=\lambda^{2} L^{T} L
$$

where $\lambda^{2}$ is a positive free parameter setting a width-scaling for the multivariate Gaussian. The inverse of the covariance matrix $C^{-1}$ is often called the Fisher information matrix. Equation 9 allows us to write equation 8 as

$$
\operatorname{prob}(F) \propto \exp \left(-\frac{1}{2} \lambda^{2}\left\|L\left(F-F_{0}\right)\right\|_{2}^{2}\right)
$$


According to Bayes' theorem (equation 5), the posterior becomes

$$
\operatorname{prob}(F \mid S) \propto \exp \left(-\frac{1}{2}\|W F-S\|_{2}^{2}-\frac{1}{2} \lambda^{2}\left\|L\left(F-F_{0}\right)\right\|_{2}^{2}\right)
$$

where we dropped the evidence term since it just scales the results which does not change the proportionality relation. Maximizing the posterior probability is equivalent to solving the minimization problem

$$
\operatorname{minimize}\left(\|W F-S\|_{2}^{2}+\lambda^{2}\left\|L\left(F-F_{0}\right)\right\|_{2}^{2}\right)
$$

We prefer the equivalent formulation as

$$
\operatorname{minimize}\left\|\left(\begin{array}{c}
W \\
\lambda L
\end{array}\right) F-\left(\begin{array}{c}
S \\
\lambda L F_{0}
\end{array}\right)\right\|_{2}
$$

as it is a stable and convenient form for numeric computation. We have hence recovered the Tikhonov problem from the Bayesian formulation. Equation 9 shows how to obtain a Fisher information matrix or its inverse, the covariance matrix $C$, from the penalty matrix $L$ in Tikhonov's problem. Conversely, given $C$, we can find $L$ to within a factor $\lambda$ by symmetric matrix decomposition. Perhaps the simplest example is the zeroth-order Tikhonov problem where $L$ is the identity matrix. Then $C$ is a diagonal matrix with $1 / \lambda^{2}$ on the diagonal, so that the entries in $F$ are uncorrelated. For first-order or higher-order Tikhonov regularization, we can likewise use equation 9 to calculate $C$. The detailed mathematical form of $C$ depends on how the penalizing derivative is implemented as finite differences.

The complete Bayesian prior corresponding to the Tikhonov problem in equa- 
tion 4 is

$$
\begin{aligned}
& \operatorname{prob}(F) \propto \exp \left(-\frac{1}{2} \lambda^{2}\left(F-F_{0}\right)^{T}(\kappa L)^{T}(\kappa L)\left(F-F_{0}\right)\right) \\
& \text { subject to }\left\{\begin{array}{l}
F^{*} \geq 0 \\
F^{*}\left(v_{\| 0}, v_{\perp 0}\right)=0 .
\end{array}\right.
\end{aligned}
$$

\section{WEIGHT FUNCTIONS}

Weight functions enter the rows of the matrix $\widetilde{W}$ in equation 1 and are analogous to lines-of-sight in standard position-space tomography. They quantify how much signal per ion is measured and are hence a convenient formulation of the forward model for velocity-space tomography. The phase space is described by the velocity coordinates $\left(v_{\|}, v_{\perp}\right)$ or $(E, p)$ and the position coordinates $\mathbf{x} .\left(v_{\|}, v_{\perp}\right)$ are the velocities parallel and perpendicular to the magnetic field, and $(E, p)$ are the energy and pitch of the particle, respectively. Here pitch is defined as $p=\frac{v_{\|}}{v}$, where $v$ is the magnitude of the ion velocity. The measurable signal $s$ can be found by integration of the weight function $w$ times the fast-ion velocity distribution function $f$ over phase space ${ }^{7,22-28}$

$s\left(m_{1}, m_{2}, \phi\right)=\int_{v o l} \int_{0}^{\infty} \int_{-\infty}^{\infty} w\left(m_{1}, m_{2}, \phi, v_{\|}, v_{\perp}, \mathbf{x}\right) f\left(v_{\|}, v_{\perp}, \mathbf{x}\right) d v_{\|} d v_{\perp} d \mathbf{x}$.

$s\left(m_{1}, m_{2}, \phi\right)$ is the integrated measured signal in the interval $m_{1}<m<m_{2}$ where $\phi$ is the angle between the line-of-sight and the magnetic field. $m$ represents the units on the abscissa of the fast-ion measurements, e.g. the energy in GRS measurements or the wavelength in FIDA measurements. The units of the integrated measured signal depend on the diagnostic units. The units of $f$ in equation 15 are $\left[\mathrm{s}^{2} / \mathrm{m}^{5}\right]$. Weight functions thus show the signal per fast ion as function of the velocity coordi- 
nates in units [signal / fast ion]. They can be calculated using a forward model of a given fast-ion diagnostic. One defines a phase-space distribution function consisting of a single pixel and computes the signal as a function of the phase-space coordinates of the pixel. Formally, we can substitute a $\delta$-function describing the position of $N_{f}$ fast ions,

$$
f_{\delta}\left(v_{\|}, v_{\perp}, \mathbf{x}\right)=N_{f} \delta\left(v_{\|}-v_{\| 0}\right) \delta\left(v_{\perp}-v_{\perp 0}\right) \delta\left(\mathbf{x}-\mathbf{x}_{0}\right)
$$

into equation 15 and effect the integration. The amplitudes of weight functions at phase-space position $\left(\mathbf{x}_{0}, v_{\| 0}, v_{\perp 0}\right)$ are then

$$
w\left(m_{1}, m_{2}, \phi, v_{\| 0}, v_{\perp 0}, \mathbf{x}_{0}\right)=\frac{s\left(m_{1}, m_{2}, \phi\right)}{N_{f}}
$$

The weight functions computed with this formalism account for any physics included in the forward model. However, they neglect any spatial variations within the measurement volume. Further, the weight functions do not account for any background noise which will therefore need to be subtracted, e.g. passive FIDA light. Uncertainties of this type will be discussed in section V..

Equation 17 does not provide any insight into the peculiar shapes of the weight functions for each diagnostic. Examples of weight functions for FIDA, CTS, a neutral particle analyzer (NPA), NES and GRS are presented in figures 1 to 6 . The basic shapes of the weight functions are explained for each diagnostic by considering the underlying physics processes. ${ }^{7,24-27}$

The FIDA weight functions ${ }^{24}$ in figure 1 illustrate the excellent coverage of velocity space by the five-view FIDA diagnostic installed at ASDEX Upgrade when the measurements are analyzed together. ${ }^{10,14}$ Each FIDA view individually misses a portion of velocity space, but integrated data analysis of all five detectors together 


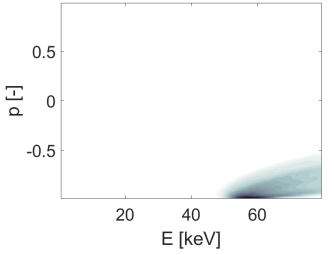

(a) $13^{\circ},-5 \mathrm{~nm}$

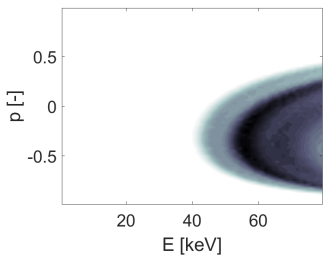

(e) $69^{\circ},-5 \mathrm{~nm}$

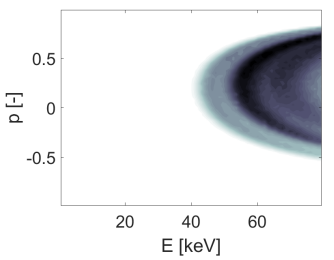

(i) $103^{\circ},-5 \mathrm{~nm}$

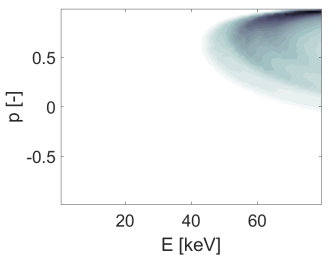

(m) $133^{\circ},-5 \mathrm{~nm}$

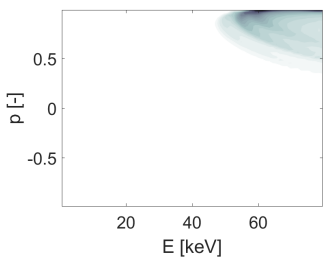

(q) $154^{\circ},-5 \mathrm{~nm}$

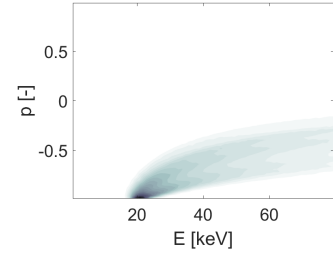

(b) $13^{\circ},-3 \mathrm{~nm}$

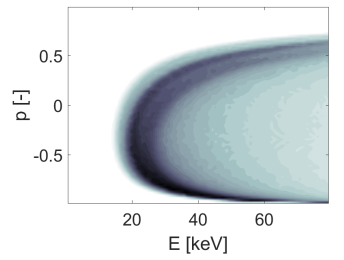

(f) $69^{\circ},-3 \mathrm{~nm}$

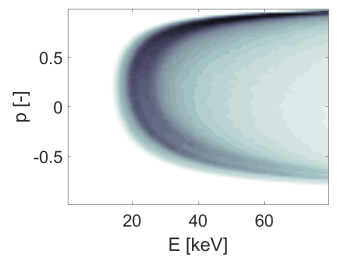

(j) $103^{\circ},-3 \mathrm{~nm}$

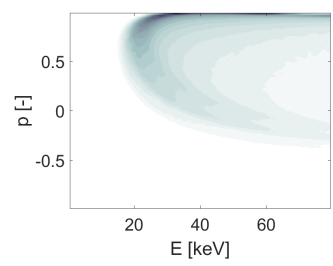

(n) $133^{\circ},-3 \mathrm{~nm}$

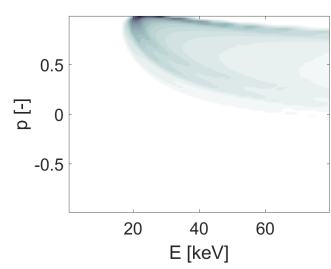

(r) $154^{\circ},-3 \mathrm{~nm}$

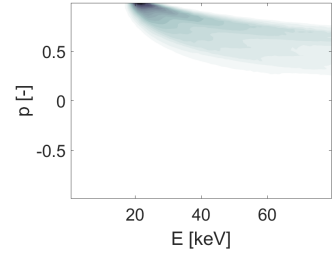

(c) $13^{\circ}, 3 \mathrm{~nm}$

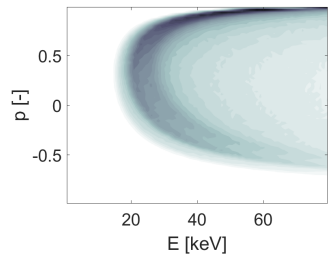

(g) $69^{\circ}, 3 \mathrm{~nm}$

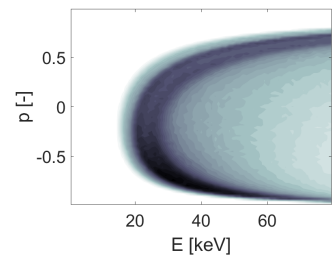

(k) $103^{\circ}, 3 \mathrm{~nm}$

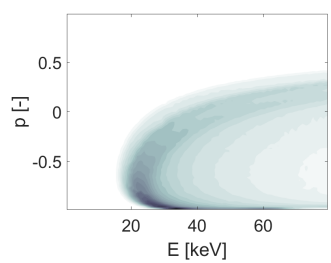

(o) $133^{\circ}, 3 \mathrm{~nm}$

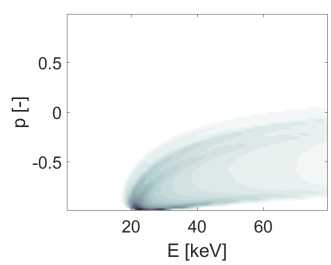

(s) $154^{\circ}, 3 \mathrm{~nm}$

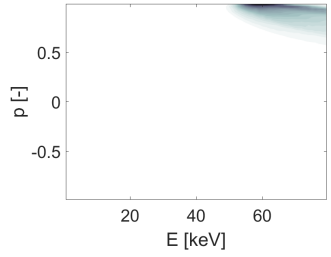

(d) $13^{\circ}, 5 \mathrm{~nm}$

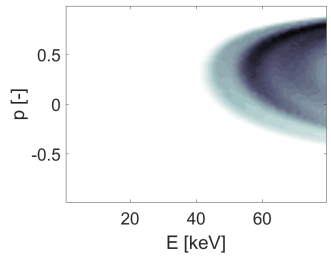

(h) $69^{\circ}, 5 \mathrm{~nm}$

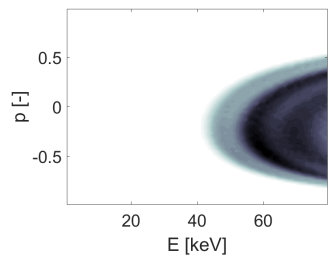

(1) $103^{\circ}, 5 \mathrm{~nm}$

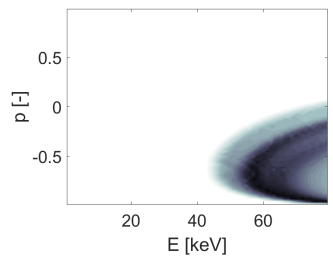

(p) $133^{\circ}, 5 \mathrm{~nm}$

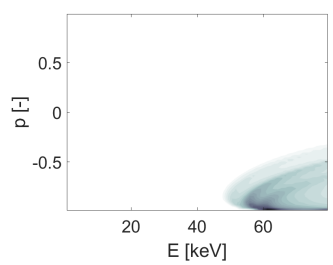

(t) $154^{\circ}, 5 \mathrm{~nm}$

Fig. 1. Typical weight functions [a.u.] for the five FIDA spectra for five different viewing angles for strong and moderate blueshifts (first and second columns) and redshifts (third and fourth columns). The angles describe the angle between the line-of-sight of the view and the local magnetic field. The wavelengths are relative to the unshifted $\mathrm{D}_{\alpha}$ radiation wavelength of $656.1 \mathrm{~nm}$. 


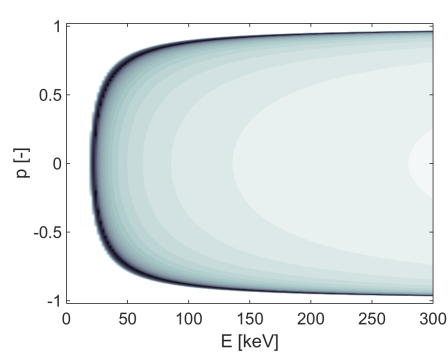

(a) $90^{\circ}, 1 \times 10^{6} \mathrm{~m} / \mathrm{s}$

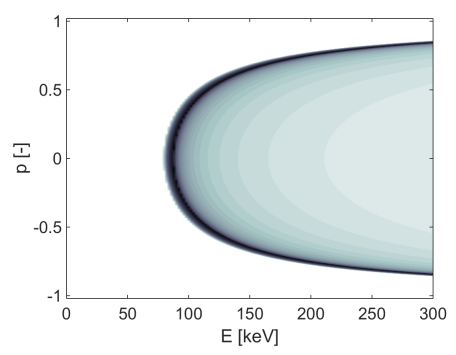

(b) $90^{\circ}, 2 \times 10^{6} \mathrm{~m} / \mathrm{s}$

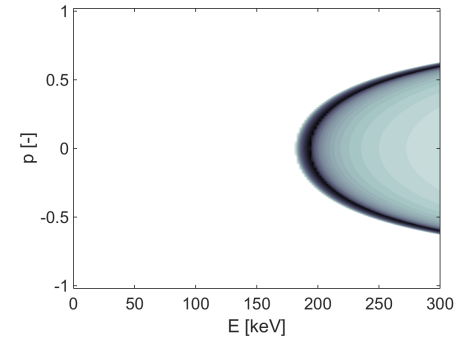

(c) $90^{\circ}, 3 \times 10^{6} \mathrm{~m} / \mathrm{s}$

Fig. 2. Typical CTS weight functions [a.u.] for a viewing angle of $90^{\circ}$ for three projected velocities.

provides excellent coverage of $2 \mathrm{D}$ velocity space allowing the measurement of velocity distribution functions. Nevertheless, the integrated data analysis can further be improved by adding additional diagnostics. In particular, the velocity-space sensitivity of FIDA drops above the beam injection energy because the charge-exchange probabilities of very highly energetic particles with beam neutrals drop for increasing particle energy. Therefore the measurements of the high-energy velocity space could likely be substantially improved by adding CTS and NES diagnostics to the FIDA tomography.

Figure 2 illustrates typical CTS weight functions for observation perpendicular to the magnetic field. ${ }^{7}$ If the geometry of CTS experiments is flexible (as often), the weight functions can take very different shapes similar to the FIDA weight functions. The overall shapes of CTS weight functions are similar to those of FIDA weight functions because both are largely determined by the Doppler shift.

Figure 3 shows weight functions for the NPA at ASDEX Upgrade. NPAs measure the energies of fast neutrals generated in charge-exchange reactions between fast ions and neutrals. The measured energy of the neutral in the detector is the same as that of the fast ion in the plasma. Ions in very small pitch and gyroangle ranges can generate a detectable neutral which is reflected in the narrow pitch range of the weight function. The width of the energy interval is the same as the chosen 


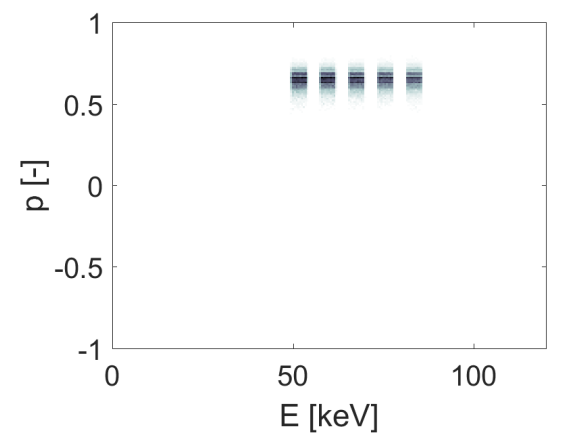

Fig. 3. Typical NPA weight functions [a.u.] for the NPA at ASDEX Upgrade for five different energy ranges of the observed neutrals which are identical to the energy ranges of the fast ions: $50-54 \mathrm{keV}, 58-62 \mathrm{keV}, 66-70 \mathrm{keV}, 74-78 \mathrm{keV}, 82-86 \mathrm{keV}$. Only narrow pitch and gyroangle ranges are accepted by the instrument.

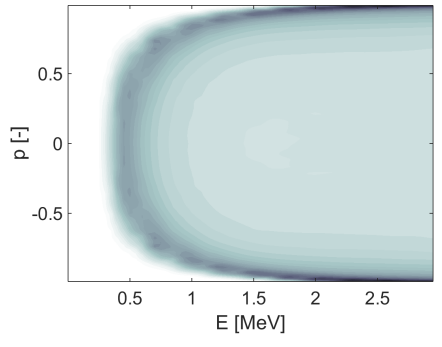

(a) $56 \mathrm{~ns}$

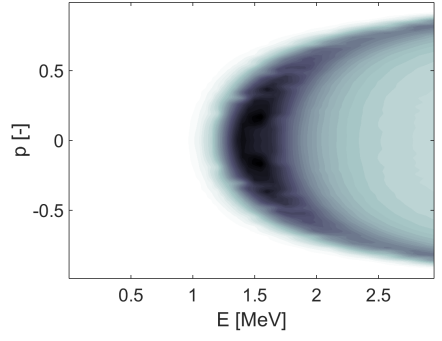

(b) $48 \mathrm{~ns}$

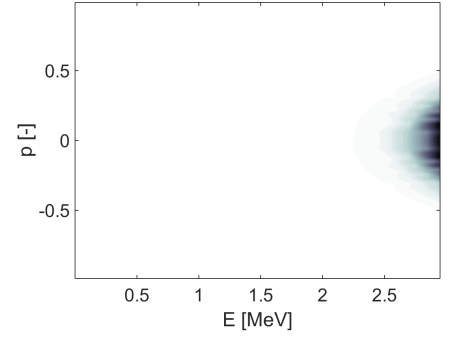

(c) $40 \mathrm{~ns}$

Fig. 4. Typical weight functions [a.u.] for TOFOR for three times-of-flight.

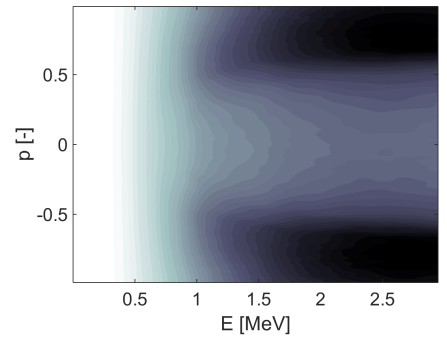

(a) $+2 \mathrm{keV}$

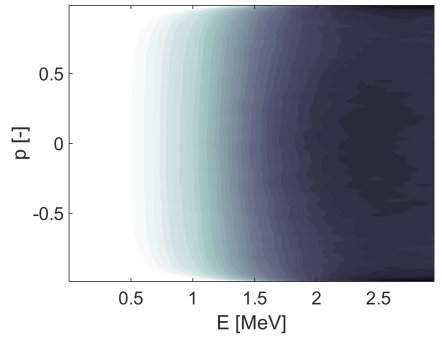

(b) $+22 \mathrm{keV}$

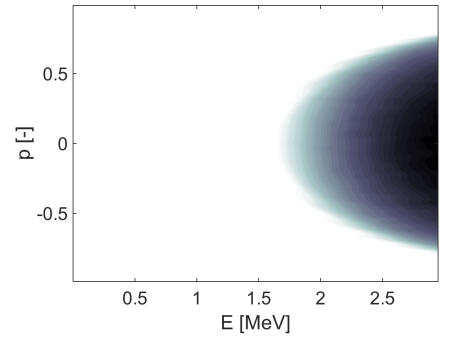

(c) $+42 \mathrm{keV}$

Fig. 5. Typical weight functions [a.u.] for two-step reaction $\gamma$-ray spectrometry using a high-purity Germanium detector resolving the nominal peak energy of $2868 \mathrm{keV}$. The shifts in (a) to (c) are relative to the nominal peak energy. 


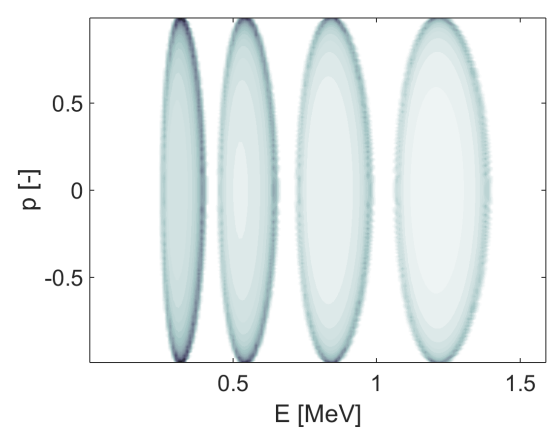

Fig. 6. Typical weight functions [a.u.] for one-step reaction $\gamma$-ray spectrometry using a high-purity Germanium detector resolving the nominal peak energy of $5500 \mathrm{keV}$. From left to right relative to the nominal peak energy: $+200 \mathrm{keV},+350 \mathrm{keV},+550 \mathrm{keV},+800 \mathrm{keV}$

energy bin width in the measurement. NPA weight functions have not yet been used in velocity-space tomography.

Figure 4 illustrates typical weight functions for the time-of-flight neutron emission spectrometer TOFOR at JET. ${ }^{25,28}$ The line-of-sight of TOFOR is approximately perpendicular to the central magnetic field at JET. ${ }^{29}$ The weight functions are calculated for the $\mathrm{D}(\mathrm{D}, \mathrm{n})^{3} \mathrm{He}$ reaction. MeV-range ions are well diagnosed by TOFOR or other NES instruments in contrast to FIDA. The low time-of-flight signals in TOFOR are not sensitive to high-energy ions with pitches close to \pm 1 (figure 4). However, NES detectors installed on oblique lines-of-sight can be sensitive to either $p \sim 1$ or $p \sim-1$.

Figure 5 shows typical weight functions for two-step reaction GRS with a HpGe detector ${ }^{26}$ here for the ${ }^{9} \mathrm{Be}(\mathrm{D}, \mathrm{n} \gamma){ }^{10} \mathrm{~B}$ reaction. An interesting feature is that at low Doppler-shift the HpGe detector is most sensitive to high-energy ions with pitches close to \pm 1 . However, at high Doppler shift these regions do not contribute any signal whereas the ions with pitches close to 0 contribute most signal. The velocityspace sensitivities of the NES and GRS detectors at JET (similar to figure 1 for FIDA) have been presented elsewhere. ${ }^{20,28}$

Lastly, figure 6 illustrates typical weight functions for one-step reaction GRS, 
here for the reaction $\mathrm{D}(\mathrm{p}, \gamma)^{3} \mathrm{He} \cdot{ }^{27,30}$ The ITER measurement requirements entail resolution of the energy spectra and densities of fusion alphas and other fast ions. ${ }^{31}$ Figure 6 illustrates that one-step reaction GRS could in principle provide direct energy resolution of the fast ions without the need for tomographic inversion, ${ }^{27}$ similar to the NPAs. The other fast-ion diagnostics have weight functions covering very broad energy ranges hampering a direct energy resolution of the individual diagnostics in principle. Tomographic inversion of all available fast-ion measurements at ITER is currently the only known way to obtain measurements of the $\alpha$-particle energy spectra and other fast ion energy spectra to fulfill the ITER measurement requirements. 31

\section{UNCERTAINTIES}

The uncertainties of velocity-space tomography are conceptually the same as for standard position-space tomography which is unfortunately not straightforward. We may divide the uncertainties into three main categories:

- Uncertainties in the fast-ion measurements $S^{9}$

- Uncertainties in the weight matrix $W$ due to uncertainties in nuisance parameters $^{12}$

- Bias uncertainties due to the regularization ${ }^{12}$

The nature of the uncertainties in the fast-ion measurements depends on the diagnostic. Usually apparently random fluctuations in the signal (so-called noise) are one contribution to the uncertainty. There can also be systematic uncertainties. An example is the passive FIDA light originating from the plasma edge rather than from the measurement volume. The passive FIDA light can be subtracted by beam modulation or passive lines-of-sight. ${ }^{23}$ Whereas uncertainties due to measurement 
noise are controlled to be small in tomography, any systematic measurement error such as in the background subtraction will propagate into the inversion. Discussions of uncertainties for various fast-ion diagnostics is found in a recent review. ${ }^{32} \mathrm{Un}$ certainties in the nuisance parameters cover the uncertainties in other parameters needed in the forward model. For example, often the densities and temperatures of ions or electrons influence the amplitude of the measured fast-ion signals. Such parameters and their uncertainties are usually measured by other diagnostics. Lastly, the bias uncertainties cover systematic errors introduced by the regularization which is needed to obtain stable solutions to the tomography problem.

Equation 3 shows that the solution in $F$ depends on the regularization parameter $\lambda$, and so do the uncertainties. For very small $\lambda$, the lower part of equation 3 becomes negligible, and one approaches the original least-squares problem. Even though the fit to the measurement data is very good, the solution is unstable and is completely dominated by measurement noise. The bias due to the regularization is in this case small. For very large $\lambda$, the upper part of equation 3 becomes negligible, and one approaches a very smooth function, but the fit to the measurement data is poor. In this case the uncertainty due to measurement noise is small, but the bias due to regularization is large. A trade-off must be made in the choice of $\lambda$ to balance the propagation of measurement noise into the inversion against systematic bias introduced by the regularization itself. Various strategies to choose $\lambda$ automatically exist, such as the L-curve ${ }^{12}$ or generalized cross-validation. ${ }^{18}$ Assuming that we have chosen $\lambda$ or choose it automatically, we can quantify the uncertainties in the inversion due to uncertainties in the fast-ion measurements and due to uncertainties in the nuisance parameters. We sample the fast-ion measurements and nuisance parameters from their probability distribution functions and compute a population 
of inversions:

$$
F_{i}^{*}=\arg \min _{F}\left\|\left(\begin{array}{c}
W_{i} \\
\lambda L
\end{array}\right) F-\left(\begin{array}{c}
S_{i} \\
0
\end{array}\right)\right\|_{2} \quad \text { subject to } F \geq 0
$$

The mean of the $N$ inversions is the best estimate of the velocity-distribution function,

$$
\left\langle F^{*}\right\rangle=\frac{1}{N} \sum_{i} F_{i} *
$$

and the uncertainty of the inversion is the corresponding standard deviation

$$
\delta F^{*}=\sqrt{\frac{1}{N-1} \sum_{i}\left(F_{i}-\left\langle F^{*}\right\rangle\right)^{2}}
$$

which accounts for uncertainties in the signal and the nuisance parameters.

Each pixel of the inversion has its own uncertainty, so that we can assess which parts of velocity space can be reliably inferred. ${ }^{12,15}$ If required, we can assess the individual contributions of the measurement noise and the nuisance parameter uncertainty by fixing the other value. The quantification of the systematic regularization bias is an open problem because we would need to know the true distribution function. If it was known, we could substitute and find the regularization bias according to

$$
\begin{aligned}
& \delta F_{\text {bias }}=\arg \min _{F}\left\|\left(\begin{array}{c}
W \\
\lambda L
\end{array}\right) F-\left(\begin{array}{c}
W F_{\text {true }} \\
0
\end{array}\right)\right\|_{2}-F_{\text {true }} \\
& \text { subject to } F \geq 0 \text {. }
\end{aligned}
$$

One may use a numerical simulation or the tomographic inversion to estimate the bias uncertainty. ${ }^{12}$ 


\section{RECONSTRUCTIONS OF A PHANTOM USING INTEGRATED DATA ANALYSIS}

In this section we demonstrate the benefits of integrated data analysis of all available fast ion measurements at JET. JET discharge \#86459 was heated by 4.5 MW NBI and $3 \mathrm{MW}$ ICRF heating at the third harmonic of deuterium. ${ }^{33} \mathrm{In}$ this scenario a strong fast-ion tail is accelerated above the NBI injection energies of $120 \mathrm{keV}$. Deuterium ions are accelerated up to energies of about $2 \mathrm{MeV}$ until they encounter the so-called barrier region in velocity space where the coupling between the wave electric field and the ions becomes very weak. ${ }^{6}$ Therefore only small populations at energies above about $2 \mathrm{MeV}$ are expected. This is shown in the ASCOT simulation in figure 7(a) where the tail terminates at about $2 \mathrm{MeV}$. The simulation also shows that the tail becomes broader towards lower energies due to collisions. A corresponding tomographic inversion of NES and GRS measurement was in excellent agreement with the simulation. ${ }^{20}$

In figure 7 we demonstrate the importance of integrated data analysis to achieve this result. The three inversions in figures $7(\mathrm{~b})$-(d) are computed using first-order Tikhonov regularization with non-negativity constraint according to equation 3 . Figure 7(b) shows an inversion from noisy, synthetic measurements using all available detectors based on the simulation in figure 7(a). The detectors are one HpGe GRS detector ${ }^{34,35}$ and three NES detectors: the time-of-flight spectrometer TOFOR ${ }^{29,36,37}$ the liquid scintillator, ${ }^{38}$ and the single crystal diamond detector. ${ }^{39-41}$ If the NES and GRS measurements are combined (figure 7(b)), the shape of the fast-ion tail is in excellent agreement with the simulation as also the inversion of actual measurements showed. ${ }^{20}$

In figure $7(\mathrm{c})$ we use only the three NES detectors. We have not been able to 
obtain inversions resembling the known solution from figure 7(a) for this case. A likely reason is that two of the three NES detectors are on an oblique line-of-sight. Detectors on this oblique line-of-sight cover only positive parallel velocities. ${ }^{20,25,28}$ The half plane with positive parallel velocities is therefore well covered by three sets of weight functions. However, the half plane with negative parallel velocities is only covered by the weight functions from TOFOR, i.e. by just one view, which is not enough for inversion.

The GRS instrument observes two $\gamma$-ray reaction peaks in high resolution, such that two sets of weight functions and independent measurements are available. These two sets cover the entire velocity space. ${ }^{20,26}$ Roughly, it is possible to localize the position of the tail, but the tail shape cannot be recovered (figure $7(d)$ ).

While TOFOR is the workhorse of this integrated data analysis, it is noteworthy that the $2 \mathrm{D}$ inversion at JET is not successful without the $\gamma$-ray measurements. With TOFOR and the HPGe detector, most of the velocity space shown in figure $7(\mathrm{~b})$ is covered by three sets of weight functions. We conclude that an essential ingredient for the velocity-space tomography method to work is that the relevant parts of velocity space are covered by more than one set of weight functions. Apparently the two sets of weight functions available from the HpGe detector are not sufficient, either.

Currently a second GRS instruments is being installed at JET which will have an oblique view $\left(\phi=30^{\circ}\right)$. In figure 8 we show an inversion based on four GRS spectra observed with two HpGe detectors whereas the NES detectors are not used. This type of all-GRS inversion is relevant for $\alpha$-particle diagnostic in the upcoming deuterium-tritium campaign as fast $\alpha$-particles do not produce neutrons directly. The reconstruction of the ASCOT simulation based on the noisy synthetic measurements (figure 8) is much improved compared with the single GRS detector measure- 


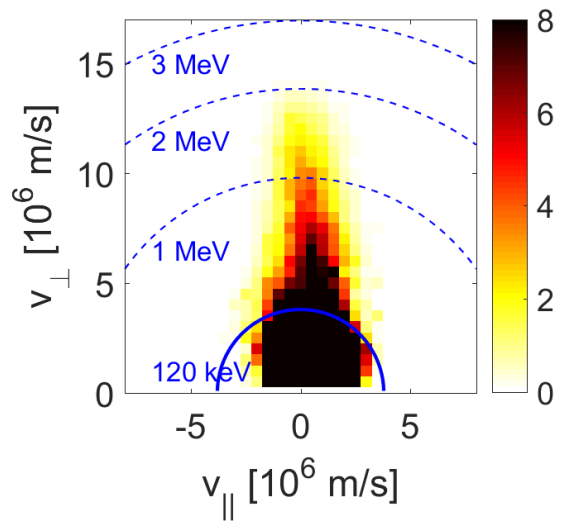

(a) ASCOT

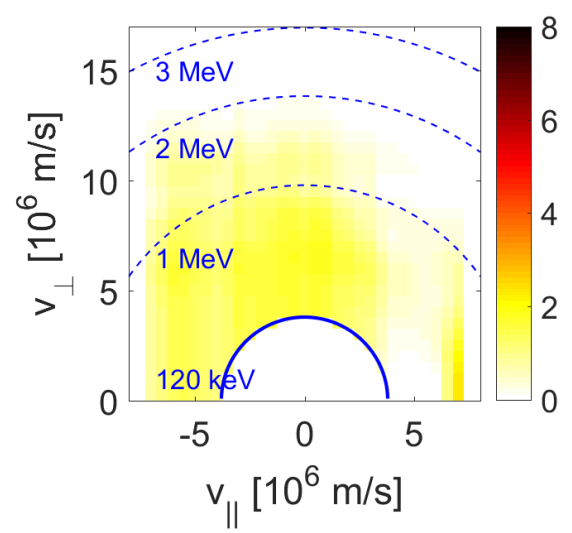

(c) NES

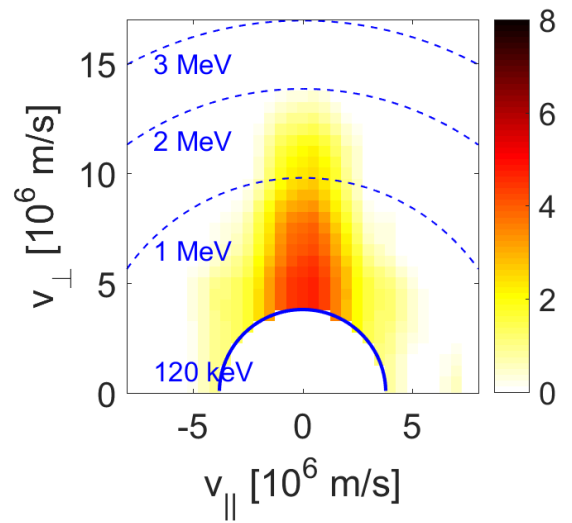

(b) NES+GRS

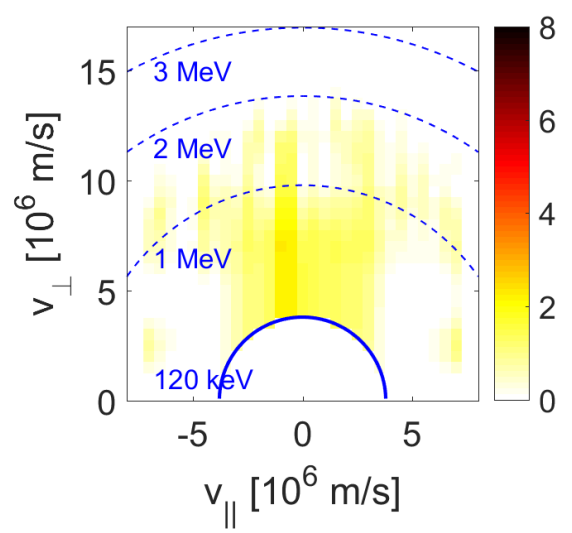

(d) GRS

Fig. 7. (a) Simulation. (b)-(d) Inversions based on noisy synthetic measurements from the ASCOT simulation. (b) NES and GRS measurements. (c) Only NES measurements. (d) Only GRS measurements. 


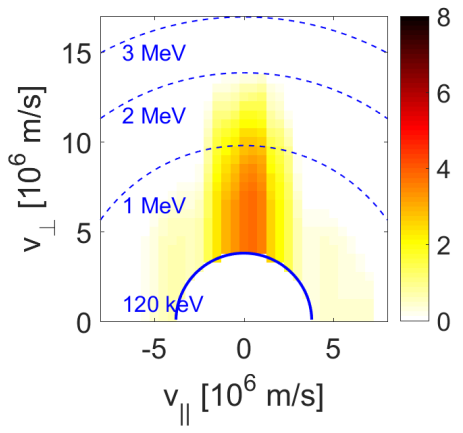

Fig. 8. Inversions based on noisy synthetic measurements from the ASCOT simulation. Here two simultaneous GRS measurements were assumed $\left(\phi_{1}=90^{\circ}, \phi_{2}=30^{\circ}\right)$

ments (figure $7(\mathrm{~d})$ ).

\section{OUTLOOK}

A near term goal of velocity-space tomography at JET and ASDEX Upgrade is to include further detectors in the integrated data analysis. ASDEX Upgrade has up to six FIDA views, ${ }^{13,42,43}$ two CTS views, ${ }^{44-46}$ one NES view, ${ }^{47,48}$ one GRS view, ${ }^{49}$ one $\mathrm{NPA}^{50}$ as well as five fast-ion loss detectors (FILD). ${ }^{51,52}$ Until now only five detectors have been used in the integrated data analysis. At JET further detectors should become available in the near future to allow measurements in the upcoming deuterium-tritium campaign. ${ }^{20,53}$ In particular, an oblique GRS view and an upgraded $\gamma$-ray camera could contribute significantly to the integrated data analysis.

Integrated data analysis based on high-definition velocity-space tomography should now be demonstrated on other machines than ASDEX Upgrade and JET. Good candidates are machines with FIDA views and additional diagnostics, such as DIII-D ${ }^{54,55}$ EAST, ${ }^{56-58}$ TCV,${ }^{59} \mathrm{NSTX}^{60}{ }^{64} \mathrm{MATT}^{61,62}$ or LHD. ${ }^{63-66}$

An important long-term goal is to measure $\alpha$-particle 2D velocity distribution functions at ITER. This would allow us to measure the $\alpha$-particle density and the $\alpha$ - 
particle energy spectrum which are part of the ITER measurement requirements. ${ }^{31}$ Core-averaged values of these parameters can be measured by integration of the measured 2D velocity-distribution function, if at least one diagnostic is absolutely calibrated. The $\alpha$-particles at ITER can be directly measured by CTS $^{67-69}$ and GRS $^{70,71}$ and possibly charge-exchange recombination spectroscopy. ${ }^{72}$ CTS measurements at ITER are being designed to be absolutely calibrated. ITER is also going to be equipped with NES. ${ }^{31}$ Deuterium and tritium $2 \mathrm{D}$ velocity distribution functions, as well as the derived fast-ion density and energy spectra, could also be determined by velocity-space tomography in ITER by CTS, NES and GRS. The various combinations of fast-ion diagnostics on the various machines provide a rich test bed to develop the velocity-space tomography method for ITER.

\section{CONCLUSIONS}

We presented an overview and current status of velocity-space tomography as an integrated data analysis tool for fast-ion measurements. As an example of an inversion method, we discussed the Tikhonov regularization and how to use various forms of prior information in the Bayesian framework. Further, we discussed weight functions and uncertainties in the inversions. The recent combined inversion of GRS and NES at JET highlights the need for integrated data analysis. Useful inversions could only be obtained by combined measurements of NES and GRS, not from NES or GRS alone. NES-only or GRS-only inversions do not even work well using synthetic data which is substantially easier than using experimental data. However, if another HpGe GRS detector with an oblique line-of-sight becomes available at JET, it could be possible to base inversions on GRS data alone. Integrated data analysis of all available fast-ion measurements by tomographic inversion appears to be a promising route to fulfil the ITER measurement requirements on the densities 
and energy spectra of $\alpha$-particles and other fast ions.

\section{ACKNOWLEDGEMENTS}

We thank the ITPA Energetic Particle Physics Topical Group for its support. This work has been carried out within the framework of the EUROfusion Consortium and has received funding from the Euratom research and training programme 20142018 under grant agreement No 633053. The views and opinions expressed herein do not necessarily reflect those of the European Commission. 


\section{REFERENCES}

${ }^{1}$ X. Litaudon et al., "Overview of the JET results in support to ITER," Nucl. Fusion, 57, 102001 (2017).

${ }^{2}$ A. Kallenbach et al., "Overview of ASDEX Upgrade results," Nucl. Fusion, 57, 102015 (2017).

${ }^{3}$ H. MEYer et al., "Overview of progress in European medium sized tokamaks towards an integrated plasma-edge/wall solution," Nucl. Fusion, 57, 102014 (2017).

${ }^{4}$ R. Fischer et al., "Bayesian modelling of fusion diagnostics," Plasma Phys. Control. Fusion, 45, 7, 1095 (2003).

${ }^{5}$ J. Svensson et al., "Integrating diagnostic data analysis for W7-AS using Bayesian graphical models," Rev. Sci. Instrum., 75, 10, 4219 (2004).

${ }^{6}$ M. SCHNEIDER et al., "Modelling third harmonic ion cyclotron acceleration of deuterium beams for JET fusion product studies experiments," Nucl. Fusion, 56, 112022 (2016).

${ }^{7}$ M. SALEWSKI et al., "On velocity space interrogation regions of fast-ion collective Thomson scattering at ITER," Nucl. Fusion, 51, 8, 083014 (2011).

${ }^{8}$ M. SALEWSKI et al., "Tomography of fast-ion velocity-space distributions from synthetic CTS and FIDA measurements," Nucl. Fusion, 52, 103008 (2012).

${ }^{9}$ M. SALEWSKI et al., "Combination of fast-ion diagnostics in velocity-space tomographies," Nucl. Fusion, 53, 063019 (2013).

${ }^{10}$ M. SALEWSKI et al., "Measurement of a 2D fast-ion velocity distribution function by tomographic inversion of fast-ion D-alpha spectra," Nucl. Fusion, 54, 023005 (2014). 
${ }^{11}$ M. SALEWSKI et al., "Doppler tomography in fusion plasmas and astrophysics," Plasma Phys. Control. Fusion, 57, 014021 (2015).

12 A. S. JACOBSEn et al., "Inversion methods for fast-ion velocity-space tomography in fusion plasmas," Plasma Phys. Control. Fusion, 58, 045016 (2016).

${ }^{13}$ B. GEIGER et al., "Fast-ion transport and neutral beam current drive in ASDEX Upgrade," Nucl. Fusion, 55, 083001 (2015).

${ }^{14}$ M. WEILAND et al., "Enhancement of the FIDA diagnostic at ASDEX Upgrade for velocity space tomography," Plasma Phys. Control. Fusion, 58, 025012 (2016).

15 A. S. JACOBSEN et al., "Benchmark and combined velocity-space tomography of fast-ion D-alpha spectroscopy and collective Thomson scattering measurements," Plasma Phys. Control. Fusion, 58, 042002 (2016).

${ }^{16}$ F. JAulmes et al., "Numerical and experimental study of the redistribution of energetic and impurity ions by sawteeth in ASDEX Upgrade," Nucl. Fusion, 56, 112012 (2016).

${ }^{17}$ J. RAsmussen et al., "Collective Thomson scattering measurements of fast-ion transport due to sawtooth crashes in ASDEX Upgrade," Nucl. Fusion, 56, 112014 (2016).

${ }^{18}$ M. SALEWSKI et al., "High-definition velocity-space tomography of fast-ion dynamics," Nucl. Fusion, 56, 106024 (2016).

${ }^{19}$ M. Weiland et al., Nucl. Fusion, Phase-space resolved measurement of 2nd harmonic ion cyclotron heating using FIDA tomography at the ASDEX Upgrade tokamak (at press). 
${ }^{20} \mathrm{M}$. SALEWSKI et al., "MeV-range velocity-space tomography from gamma-ray and neutron emission spectrometry measurements at JET," Nucl. Fusion, 57, $056001(2017)$

${ }^{21}$ U. Stroth et al., "Overview of ASDEX Upgrade results," Nucl. Fusion, 53, $104003(2013)$

${ }^{22} \mathrm{~W}$. W. HEIDBRink et al., "Measurements of fast-ion acceleration at cyclotron harmonics using Balmer-alpha spectroscopy," Plasma Phys. Control. Fusion, 49, 9, 1457 (2007).

${ }^{23}$ W. W. HeIdbrink, "Fast-ion D $\alpha$ measurements of the fast-ion distribution (invited)." Rev. Sci. Instrum., 81, 10D727 (2010).

${ }^{24}$ M. SAlEwski et al., "On velocity-space sensitivity of fast-ion D-alpha spectroscopy," Plasma Phys. Control. Fusion, 56, 105005 (2014).

${ }^{25}$ A. S. JaCOBSEn et al., "Velocity-space sensitivity of neutron spectrometry measurements," Nucl. Fusion, 55, 053013 (2015).

${ }^{26}$ M. SALEWSKI et al., "Velocity-space observation regions of high-resolution twostep reaction gamma-ray spectroscopy," Nucl. Fusion, 55, 093029 (2015).

${ }^{27}$ M. SALEWSKI et al., "Fast-ion energy resolution by one-step reaction gamma-ray spectrometry," Nucl. Fusion, 56, 046009 (2016).

${ }^{28}$ A. S. JaCobSEn et al., "Velocity-space sensitivities of neutron emission spectrometers at JET and ASDEX Upgrade," Rev. Sci. Instrum., 88, 073506 (2017).

${ }^{29}$ M. Gatu Johnson et al., "The 2.5-MeV neutron time-of-flight spectrometer TOFOR for experiments at JET," Nucl. Inst. Meth. Phys. Res. A, 591, 417 (2008). 
${ }^{30}$ M. Nocente et al., "Gamma-ray emission spectrum from thermonuclear fusion reactions without intrinsic broadening," Nucl. Fusion, 55, 123009 (2015).

${ }^{31}$ A. J. H. Donné et al., "Progress in the ITER Physics Basis Chapter 7: Diagnostics," Nucl. Fusion, 47, 6, S337 (2007).

32 D. Moseev et al., Reviews of Modern Plasma Physics, Fast ion diagnostics in fusion plasmas (submitted).

${ }^{33}$ J. ERIKSSON et al., "Dual sightline measurements of MeV range deuterons with neutron and gamma-ray spectroscopy at JET," Nucl. Fusion, 55, 123026 (2015).

${ }^{34}$ M. NocENTE et al., "Energy resolution of gamma-ray spectroscopy of JET plasmas with a LaBr[sub 3] scintillator detector and digital data acquisition," Rev. Sci. Instrum., 81, 10D321 (2010).

${ }^{35}$ M. Nocente et al., "High resolution gamma ray spectroscopy at $\mathrm{MHz}$ counting rates with LaBr 3 Scintillators for fusion plasma applications," IEEE Trans. Nucl. Sci., 60, 2, 1408 (2013).

${ }^{36}$ C. Hellesen et al, "Measurements of fast ions and their interactions with MHD activity using neutron emission spectroscopy," Nucl. Fusion, 50, 084006 (2010).

${ }^{37}$ C. Hellesen et al, "Fast-ion distributions from third harmonic ICRF heating studied with neutron emission spectroscopy," Nucl. Fusion, 53, 113009 (2013).

${ }^{38}$ F. BINDA et al., "Forward fitting of experimental data from a NE213 neutron detector installed with the magnetic proton recoil upgraded spectrometer at JETa)," Rev. Sci. Instrum., 85, 11E123 (2014).

${ }^{39}$ C. CAzzaniga et al., "Single crystal diamond detector measurements of deuterium-deuterium and deuterium-tritium neutrons in Joint European Torus fusion plasmas," Rev. Sci. Instruments, 85, 043506 (2014). 
${ }^{40}$ M. Nocente et al., "Fast ion energy distribution from third harmonic radio frequency heating measured with a single crystal diamond detector at the Joint European Torus," Rev. Sci. Instruments, 86, 103501 (2015).

${ }^{41}$ A. Muraro et al., "First neutron spectroscopy measurements with a pixelated diamond detector at JET," Rev. Sci. Instruments, 87, 11D833 (2016).

${ }^{42}$ B. Geiger et al., "Multi-view fast-ion D-alpha spectroscopy diagnostic at ASDEX Upgrade." Rev. Sci. Instrum., 84, 113502 (2013).

${ }^{43}$ B. Geiger et al., "Quantification of the impact of large and small-scale instabilities on the fast-ion confinement in ASDEX Upgrade," Plasma Phys. Control. Fusion, 57, 014018 (2015).

${ }^{44}$ M. SALEWSKI et al., "Comparison of fast ion collective Thomson scattering measurements at ASDEX Upgrade with numerical simulations," Nucl. Fusion, 50, $035012(2010)$

${ }^{45} \mathrm{~S}$. K. NIELSEN et al., "Measurements of the fast-ion distribution function at ASDEX upgrade by collective Thomson scattering (CTS) using active and passive views," Plasma Phys. Control. Fusion, 57, 035009 (2015).

46 J. RASMUSSEN et al., "Consistency between real and synthetic fast-ion measurements at ASDEX Upgrade," Plasma Phys. Control. Fusion, 57, 075014 (2015).

${ }^{47} \mathrm{G}$. TARDINI et al., "First neutron spectrometry measurements in the ASDEX Upgrade tokamak," J. Instrum., 7, C03004 (2012).

${ }^{48} \mathrm{G}$. TARDINI et al., "Simulation of the neutron rate in ASDEX Upgrade H-mode discharges," Nucl. Fusion, 53, 063027 (2013).

${ }^{49}$ M. Nocente et al., "Gamma-ray spectroscopy measurements of confined fast ions on ASDEX Upgrade," Nucl. Fusion, 52, 094021 (2012). 
${ }^{50} \mathrm{P}$. A. SCHNEIDER et al., "A new compact solid-state neutral particle analyser at ASDEX Upgrade: Setup and physics modeling." Rev. Sci. Instrum., 86, 073508 $(2015)$

${ }^{51}$ M. Garcia-Munoz et al., "Fast-ion redistribution and loss due to edge perturbations in the ASDEX Upgrade, DIII-D and KSTAR tokamaks," Nucl. Fusion, 53, 123008 (2013).

${ }^{52}$ M. Garcia-Munoz et al., "Fast-ion losses induced by ELMs and externally applied magnetic perturbations in the ASDEX Upgrade tokamak," Plasma Phys. Control. Fusion, 55, 124014 (2013).

${ }^{53}$ F. Romanelli, "Overview of the JET results," Nucl. Fusion, 55, 104001 (2015).

${ }^{54} \mathrm{~W}$. W. HeIdBrink et al., "Hydrogenic fast-ion diagnostic using Balmer-alpha light," Plasma Phys. Control. Fusion, 46, 12, 1855 (2004).

${ }^{55}$ W. W. HeidBrink et al., "Confinement degradation by Alfvén-eigenmode induced fast-ion transport in steady-state scenario discharges," Plasma Phys. Control. Fusion, 56, 095030 (2014).

${ }^{56} \mathrm{X}$. YUAN et al., "Neutron energy spectrum measurements with a compact liquid scintillation detector on EAST," J. Instrum., 8, P07016 (2013).

${ }^{57} \mathrm{X}$. ZhANG et al., "Diagnosing NB plasmas on the EAST tokamak with new timeof-flight neutron spectrometer," Nuclear Fusion, 54, 104008 (2014).

${ }^{58}$ J. HuANG et al, "Conceptual design of a fast-ion D-alpha diagnostic on experimental advanced superconducting tokamaka)," Rev. Sci. Instrum., 85, 11E407 (2014). 
${ }^{59}$ B. Geiger et al., Plasma Phys. Control. Fusion, Study of the fast-ion distribution function in the TCV tokamak based on FIDA spectroscopy and the TRANSP code (2017).

${ }^{60}$ A. Bortolon et al., "A tangentially viewing fast ion D-alpha diagnostic for NSTX." Rev. Sci. Instrum., 81, 10D728 (2010).

${ }^{61}$ C. A. Michael et al., "Dual view FIDA measurements on MAST," Plasma Phys. Control. Fusion, 55, 095007 (2013).

${ }^{62}$ O. M. Jones et al., "Fast-ion deuterium alpha spectroscopic observations of the effects of fishbones in the Mega-Ampere Spherical Tokamak," Plasma Phys. Control. Fusion, 55, 085009 (2013).

${ }^{63} \mathrm{M}$. Nishiura et al, "Spectrum response and analysis of $77 \mathrm{GHz}$ band collective Thomson scattering diagnostic for bulk and fast ions in LHD plasmas," Nucl. Fusion, 54, 023006 (2014).

${ }^{64} \mathrm{~T}$. ITO et al, "Fast ion charge exchange spectroscopy adapted for tangential viewing geometry in LHD." Rev. Sci. Instrum., 81, 10D327 (2010).

${ }^{65}$ T. Iто et al, "Effect of Halo Neutrals on Fast-Ion Charge Exchange Spectroscopy Measurements in LHD," Plasma Fusion Res., 5, S2099 (2012).

${ }^{66} \mathrm{H}$. TOMita et al., "Development of neutron spectrometer toward deuterium plasma diagnostics in LHD," Rev. Sci. Instrum., 81, 10 D309 (2010).

${ }^{67}$ M. SALEWSKI et al., "Investigation of first mirror heating for the collective Thomson scattering diagnostic in ITER." Rev. Sci. Instrum., 79, 10E729 (2008).

${ }^{68}$ M. SALEWSKI et al., "Comparison of collective Thomson scattering signals due to fast ions in ITER scenarios with fusion and auxiliary heating," Plasma Phys. Control. Fusion, 51, 035006 (2009). 
${ }^{69}$ M. SALEWSKI et al., "Impact of ICRH on the measurement of fusion alphas by collective Thomson scattering in ITER," Nucl. Fusion, 49, 025006 (2009).

${ }^{70}$ I. N. Chugunov et al., "Development of gamma-ray diagnostics for ITER," Nucl. Fusion, 51, 083010 (2011).

${ }^{71}$ M. NocEnTE et al., "Conceptual design of the radial gamma ray spectrometers system for alpha-particle and runaway electron measurements at ITER," Nucl. Fusion, 57, 076016 (2017).

${ }^{72}$ A. Kappatou et al., "Feasibility of non-thermal helium measurements with charge exchange spectroscopy on ITER," Nucl. Fusion, 52, 043007 (2012). 